\title{
Extrato alcoólico de própolis no controle de verminoses em ovinos
}

\author{
Alcoholic extract of propolis for worms control in sheep \\ Extracto alcohólico de propóleo en el control de verminosas en ovejas
}

Recebido: 15/09/2021 | Revisado: 25/09/2021 | Aceito: 16/12/2021 | Publicado: 01/01/2022

\author{
Marlus Linécio \\ ORCID: https://orcid.org/0000-0002-9501-3810 \\ Universidade Estadual do Oeste do Paraná, Brasil \\ E-mail: marlusveterinario@gmail.com \\ Regina Conceição Garcia \\ ORCID: https://orcid.org/0000-0002-6120-5849 \\ Universidade Estadual do Oeste do Paraná, Brasil \\ E-mail: garcia.regina8@gmail.com \\ Douglas Galhardo \\ ORCID: https://orcid.org/0000-0002-2707-9076 \\ Universidade Estadual de Maringá, Brasil \\ E-mail: galhardo.douglas@gmail.com \\ Newton Tavares Escocard de Oliveira \\ ORCID: https://orcid.org/0000-0003-3347-9399 \\ Universidade Estadual do Oeste do Paraná, Brasil \\ E-mail: newtonescocard@ hotmail.com.br \\ Erika Cosendey Toledo de Mello Peixoto \\ ORCID: https://orcid.org/0000-0002-9608-4282 \\ Universidade Estadual do Norte do Paraná, Brasil \\ E-mail: emellopeixoto@uenp.edu.br
}

\begin{abstract}
Resumo
Objetivou-se avaliar o efeito antiparasitário de diferentes dosagens do extrato alcoólico da própolis no controle de helmintos gastrointestinais em ovinos Santa Inês. Foram utilizadas 32 ovelhas da raça Santa Inês, distribuídas em delineamento experimental de blocos completos casualizados, com quatro tratamentos e oito repetições. Os tratamentos foram: $10 \mathrm{~mL}$ de água; $10 \mathrm{~mL}$ de álcool 70\%; $5 \mathrm{~mL}$ de extrato alcoólico de própolis (EAP) a $30 \%$ e 10 $\mathrm{mL}$ de EAP $30 \%$. Os tratamentos foram administrados por via oral, em dose única. Os efeitos dos tratamentos foram avaliados pela contagem de ovos por gramas de fezes (OPG), referentes aos 1, 3, 7 e 21 dias após a administração. As administrações orais de $10 \mathrm{~mL}$ de água ou $10 \mathrm{~mL}$ de álcool $70 \%$ não demonstraram efeito na redução da OPG. A utilização de $5 \mathrm{~mL}$ de EAP 30\% reduziu a OPG nos dias 3 e 7, reduzindo sua eficácia no dia 21, enquanto a utilização de $10 \mathrm{~mL}$ de EAP 30\% reduziu a OPG em todos os dias avaliados, ambas por via oral. Desse modo, a administração via oral, em dose única, de $10 \mathrm{~mL}$ de extrato alcoólico da própolis a $30 \%$, reduziu de maneira mais efetiva a número de OPG, demonstrando efeito antiparasitário, podendo ser utilizada no controle de verminoses como um método alternativo aos produtos químicos sintéticos.
\end{abstract}

Palavras-chave: Antiparasitário; Segurança alimentar; Sanidade; Santa Inês; Vermes.

\begin{abstract}
The objective was to evaluate the antiparasitic effect of different dosages of the alcoholic extract of propolis in the control of verminosis in sheep. Thirty-two Santa Inês sheep were used in a randomized complete block design with four treatments and eight replicates. The treatments were: $10 \mathrm{~mL}$ of water; $10 \mathrm{~mL}$ of alcohol $70 \% ; 5 \mathrm{~mL}$ of propolis alcoholic extract (PAE) 30\%; and $10 \mathrm{~mL}$ of PAE 30\%. The treatments were administered orally, in a single dose. The effects of the treatments were evaluated by counting eggs per gram of faeces (EPG) for days $1 ; 3 ; 7$ and 21 after administration. Oral administration of $10 \mathrm{~mL}$ of water or $10 \mathrm{~mL}$ of alcohol $70 \%$ had no effect on EPG reduction. The use of $5 \mathrm{~mL}$ of PAE 30\% reduced EPG on days 3 and 7, reducing its efficacy on day 21, while the use of $10 \mathrm{~mL}$ of PAE $30 \%$ reduced EPG on all evaluated days. Thus, oral administration, in a single dose, of $10 \mathrm{~mL}$ of propolis alcoholic extract $30 \%$, effectively reduced the number of EPG demonstrating antiparasitic effect and can be used in the control of verminoses as an alternative method to chemical products synthetic.
\end{abstract}

Keywords: Antiparasitic; Food safety; Sanitation; Santa Inês; Worms.

\section{Resumen}

Objetivo fue evaluar el efecto antiparasitario de diferentes dosis de extracto alcohólico de propóleo en el control de helmintos gastrointestinales en ovejas Santa Inês. Se utilizaron 32 ovejas Santa Inês, distribuidas en un diseño experimental en bloques completos al azar, con cuatro tratamientos y ocho repeticiones. Los tratamientos fueron: 10 $\mathrm{mL}$ de agua; $10 \mathrm{~mL}$ de alcohol al 70\%; $5 \mathrm{~mL}$ de extracto alcohólico de propóleo (EAP) al 30\% y $10 \mathrm{~mL}$ de EAP al 
$30 \%$. Los tratamientos se administraron por vía oral, en una sola dosis. Los efectos de los tratamientos se evaluaron contando huevos por gramo de heces (OPG), referidos a 1, 3, 7 y 21 días después de la administración. Las administraciones orales de $10 \mathrm{~mL}$ de agua o $10 \mathrm{~mL}$ de alcohol al $70 \%$ no mostraron ningún efecto en la reducción de OPG. El uso de $5 \mathrm{~mL}$ de EAP al 30\% redujo la OPG los días 3 y 7, reduciendo su efectividad el día 21, mientras que el uso de $10 \mathrm{~mL}$ de EAP al 30\% redujo la OPG en todos los días evaluados, ambos por vía oral. Así, la administración oral, en una sola dosis, de $10 \mathrm{~mL}$ de extracto alcohólico de propóleo al 30\%, redujo de manera más efectiva el número de OPG, demostrando un efecto antiparasitario, pudiendo ser utilizado en el control de la verminosis como método alternativo para productos químicos sintéticos.

Palabras clave: Antiparasitario; Seguridad alimenticia; Cordura; Santa Inês; Gusanos.

\section{Introdução}

A criação de ovinos é uma atividade crescente no Brasil e encontra-se distribuída por todas as regiões do país. No ano de 2018, o rebanho efetivo de ovinos atingiu 18,95 milhões de cabeças, sendo que $67 \%$ desse total encontra-se na região nordeste e $21 \%$ na região sul (IBGE, 2018). O sucesso na produção de ovinos, assim como de qualquer outra produção animal, depende de garantir ao rebanho uma boa nutrição, sanidade e bem-estar animal (Sargison, 2020).

Dentre os vários desafios da criação, o controle eficiente de verminoses merece atenção, uma vez que essa espécie é amplamente acometida por esses parasitas. As verminoses ameaçam a produtividade animal e a produção de alimentos, causando perdas econômicas que atingem mais de três bilhões de dólares por ano no mundo (Salkova et al., 2015).

O controle químico desses parasitas através do uso de anti-helmínticos, foi durante muito tempo uma prática amplamente recomendada para reduzir a carga parasitária e otimizar a produção animal (Kenyon et al., 2017). Todavia, o uso indiscriminado de antibióticos, subdosagens, diagnósticos incorretos e falta de rotatividade de princípios ativos, têm provocado resistência dos parasitas a esses químicos (Goncuoglu et al., 2010; Gharsa et al., 2012; Shabana \& Al-nazi, 2020) e o risco de transferência de resíduos para os produtos animais (Morsy et al., 2013). Desse modo, há uma necessidade de estudos de novas substâncias com capacidade anti-helmíntica e de caráter natural, que possam reduzir a resistência dos parasitas, a poluição ambiental e os resíduos dos produtos (Heinzen et al., 2012; Santos et al., 2019).

A própolis é uma dessas substâncias naturais com potencial de utilização, caracterizada como um medicamento natural e não tóxico, quando administrada corretamente (Machado et al., 2016). A própolis é uma resina utilizada para vedação das colônias, produzida pelas abelhas a partir de exsudados de plantas, misturados com as secreções salivares, cera e pólen (Lima et al., 2015). Dentre os diversos componentes químicos da própolis destacam-se os compostos fenólicos e substâncias flavonoides (Coelho et al., 2017; Monroy et al., 2018), que possuem ação antiparasitária (Heinzen et al., 2012; Buck et al., 2017). Na nutrição animal, a suplementação com própolis melhorou a produção e a composição do leite, assim como as funções imunológicas em ovelhas, houve efeito no desempenho de crescimento e status antioxidante em cordeiros em condições áridas (Shedeed et al., 2019).

Todavia, as propriedades terapêuticas da própolis são variáveis uma vez que essa depende da composição florística e ecológica do ambiente no momento da coleta pelas abelhas (Morsy et al., 2013; Calegari et al., 2017). Atualmente, no Brasil, existem 13 tipos de própolis, de acordo com sua composição físico-química, origem botânica e área geográfica que a mesma foi coletada (Sena-Lopes et al., 2018). Desse modo, fazem-se necessários estudos para definir o potencial de utilização de cada um dos tipos existentes. Assim, objetivou-se avaliar o efeito antiparasitário de diferentes dosagens do extrato alcoólico da própolis no controle de verminose de ovelhas da raça Santa Inês, naturalmente infectadas. 


\section{Metodologia}

\subsection{Local, delineamento experimental e tratamentos}

O experimento foi realizado em uma propriedade localizada no município de Marechal Cândido Rondon, Paraná, situada a uma latitude $24^{\circ} 33^{\prime} 40^{\prime \prime} \mathrm{S}$, longitude $54^{\circ} 04^{\prime} 12^{\prime \prime} \mathrm{W}$ e $420 \mathrm{~m}$ de altitude entre os meses de outubro e novembro. O clima da região, segundo a classificação de Köppen, é subtropical úmido, com verões quentes e chuvas bem distribuídas.

Foram utilizadas 32 ovelhas da raça Santa Inês, não prenhas, com peso médio de 21,73 kg e idade média de dois anos. Os animais foram distribuídos em delineamento experimental de blocos completos casualizados, de acordo com a carga parasitária total inicial, com quatro tratamentos e oito repetições. Os tratamentos testados foram: $10 \mathrm{~mL}$ de água (Controle); 10 $\mathrm{mL}$ de álcool (70\% p/p); $5 \mathrm{~mL}$ de extrato alcoólico de própolis (EAP) a 30\%; e $10 \mathrm{~mL}$ de EAP a 30\%, sendo todos administrados em dose única.

A própolis bruta utilizada no preparo do extrato alcoólico da própolis foi obtida junto à COOFAMEL (Cooperativa Agrofamiliar Solidária de Apicultores do Oeste do Paraná), sendo composta de sub-amostras provenientes de produtores apícolas da região de Marechal Cândido Rondon. Para obtenção do EAP a 30\% foi utilizado álcool (70\%) como solvente e essa proporção foi estimada em função do peso da própolis em relação ao peso do álcool. A mistura foi acondicionada em frascos de coloração âmbar, que foram mantidos ao abrigo da luz e agitados diariamente durante 10 dias consecutivos (Garcia et al., 2004).

\subsection{Manejo dos animais e procedimentos de coletas}

Os animais foram confinados em 16 baias de $3 \times 3$ metros, totalizando nove metros quadrados, com dois animais por baia, em aprisco com piso de chão batido e divisórias de alvenaria, que impediam o contato entre os animais de outras baias. Durante o experimento os animais receberam feno de Lolium multiflorum e água ad libitum.

Todos os animais utilizados estavam a um período mínimo de dez meses sem qualquer tipo de tratamento parasitológico. Primeiramente, os animais passaram por um período de adaptação de 14 dias, seguido de um período experimental de 21 dias. Os animais foram distribuídos em blocos, a partir da carga parasitária que apresentavam. Inicialmente foi realizada uma coleta de fezes, antes do período de adaptação, para quantificar o número de ovos por gramas de fezes (OPG). No dia anterior às aplicações dos tratamentos foi realizada outra coleta, para confirmar e ordenar os animais em seus blocos. Algumas horas antes da aplicação dos tratamentos, realizou-se outra coleta de fezes, a fim de atualizar os valores de OPG. Todas as amostras foram coletadas individualmente, diretamente da ampola retal, por meio de sacos plásticos, e refrigeradas, a aproximadamente $4^{\circ} \mathrm{C}$.

Os tratamentos foram administrados utilizando-se seringa plástica descartável, por via oral, em dose única, de acordo com os tratamentos. Após a aplicação dos tratamentos (dia 0), foram realizadas novas coletas, nos dias 1, 3, 7 e 21, a fim de quantificar o número OPG, pela técnica de McMaster modificada (Gordon \& Whitlock, 1939). Todas as análises laboratoriais foram realizadas no dia posterior às coletas e em triplicata.

\subsection{Procedimentos estatísticos}

O efeito da redução no número de OPG no decorrer do tempo foi estimado pela comparação das amostras de OPG, após o uso dos tratamentos, com a amostra de OPG inicial (0 dia). Utilizou-se o teste pareado de Wilcoxon para estabelecer os contrastes entre as diferenças de OPG inicial em relação às OPG do $1^{\circ}$ dia (OPGD1), $3^{\circ}$ dia (OPGD3), $7^{\circ}$ dia (OPGD7) e $21^{\circ}$ dia (OPG21D) após a aplicação dos tratamentos. Os efeitos dos tratamentos foram testados por meio da análise não 
paramétrica de Mann-Whitney (Snedecor \& Cochran, 1989), que compara independentemente duas somas de escore de tratamentos $\left(\mathrm{H} 0: \sum \mathrm{Ri}=\sum \mathrm{Ri}^{\prime}\right)$. A significância foi declarada quando $\mathrm{P}<0,05$ e a tendência quando $0,10<\mathrm{P}>0,05$.

\section{Resultados}

A administração oral de $10 \mathrm{~mL}$ de água ou de álcool $70 \%$ não afeta na redução do número de ovos por grama de fezes, independente do dia avaliado (Tabela 1). A utilização de $5 \mathrm{~mL}$ de EAP $30 \%$ não reduziu a OPG após $1^{\circ}$ dia a administração desse tratamento, no entanto, ocorrer uma redução aos $3^{\circ}, 7^{\circ}$ e $21^{\circ}$ dias. A dosagem de $10 \mathrm{~mL}$ do EAP a $30 \%$, reduziu a $\mathrm{OPG}$ em todos os dias reduzidos $\left(1^{\circ}, 3^{\circ}, 7^{\circ}\right.$ e $21^{\circ}$ dias), em relação a OPG inicial (dia 0). Assim, a maior dosagem do EAP foi mais eficiente no combate aos helmintos totais em relação aos tratamentos demais, sendo todos realizados em administração oral única.

Tabela 1 - Efeito da administração oral de diferentes dosagens de extrato alcoólico de própolis (EAP) na redução do número de ovos por grama (OPG) de fezes de ovelhas Santa Inês.

\begin{tabular}{|c|c|c|c|c|}
\hline Característica $^{1}$ & $\mathrm{~N}$ & $\mathrm{~N}$ do teste & Estatística calculada $(\mathrm{W})^{2}$ & Probabilidade $^{3}$ \\
\hline \multicolumn{5}{|l|}{$10 \mathrm{~mL}$ de água } \\
\hline OPG1D & 8 & 6 & 1,5 & 0,6563 \\
\hline OPG3D & 7 & 2 & $-0,5$ & 0,5000 \\
\hline OPG7D & 7 & 6 & 4,5 & 0,8438 \\
\hline OPG21D & 7 & 7 & 7,0 & 0,8906 \\
\hline \multicolumn{5}{|c|}{$10 \mathrm{~mL}$ de álcool $70 \%$} \\
\hline OPG1D & 8 & 5 & 5,0 & 0,9063 \\
\hline OPG3D & 7 & 6 & 3,5 & 0,7813 \\
\hline OPG7D & 7 & 5 & 7,5 & 1,0000 \\
\hline OPG21D & 7 & 6 & 9,0 & 0,9688 \\
\hline \multicolumn{5}{|c|}{$5 \mathrm{~mL}$ de EAP $30 \%$} \\
\hline OPG1D & 8 & 6 & $-4,5$ & 0,2188 \\
\hline OPG3D & 8 & 7 & -14 & 0,0078 \\
\hline OPG7D & 8 & 8 & -15 & 0,0195 \\
\hline OPG21D & 8 & 8 & -12 & 0,0547 \\
\hline \multicolumn{5}{|c|}{$10 \mathrm{~mL}$ de EAP $30 \%$} \\
\hline OPG1D & 8 & 7 & -14 & 0,0078 \\
\hline OPG3D & 8 & 8 & -18 & 0,0039 \\
\hline OPG7D & 8 & 8 & -18 & 0,0039 \\
\hline OPG21D & 8 & 8 & -18 & 0,0039 \\
\hline
\end{tabular}

${ }^{1}$ OPG1D, OPG3D, OPG7D e OPG21D = diferença entre o número de ovos por grama (OPG) das fezes: 1 dia, 3 dias, 7 dias e 21 dias, respectivamente, após a administração dos tratamentos em relação ao OPG inicial; ${ }^{2}$ Soma dos postos positivos $\left(\mathrm{R}_{\mathrm{i}}^{+}\right)$menos o valor médio $\left(\mathrm{R}_{\mathrm{i}}^{+}-\mathrm{n}(\mathrm{n}+1) / 4\right)$; ${ }^{3}$ Probabilidade de significância pelo teste dos postos sinalizados de Wilcoxon, com hipótese alternativa unilateral à esquerda. Fonte: Autores.

Em relação à eficácia obtida no presente estudo, com relação ao período de atuação de cada tratamento, as probabilidades (P-value) dos escores de OPG, referentes ao $1^{\circ}$ dia após a administração dos tratamentos (OPG1) indicou semelhanças quando comparados água com álcool, água com $5 \mathrm{~mL}$ de EAP, álcool com $5 \mathrm{~mL}$ de EAP e $5 \mathrm{~mL}$ de EAP com 10 $\mathrm{mL}$ de EAP (Tabela 2). A OPG obtida nas fezes dos animais tratados com $5 \mathrm{~mL}$ de EAP observou uma redução em relação aos animais tratados com água e álcool, porém não significativo para escore do primeiro dia ( $\mathrm{P}=0,0670)$, porém houve diferença no escore do terceiro dia após a aplicação ( $\mathrm{P}=0,0065)$. Observou-se redução na OPG, quando o tratamento com $10 \mathrm{~mL}$ de EAP foi comparado com o tratamento controle (água e álcool 70\%), confirmando que a maior dosagem de EAP foi mais efetiva sobre a redução de OPG nas primeiras 24 horas após sua administração. Em relação ao $3^{\circ}$ dia após os animais serem tratados, a OPG reduziu com a utilização de $5 \mathrm{~mL}$ ou $10 \mathrm{~mL}$ de EAP 30\%, em comparação a água ou ao álcool (Tabela 2). 
Tabela 2 - Comparativo da diferença de escore do número inicial de ovos por grama (OPG) de fezes de ovinos em relação ao número de OPG do $1^{\circ}$ e do $3^{\circ}$ dia após a administração de cada tratamento.

\begin{tabular}{|c|c|c|c|c|}
\hline Tratamento & $\mathrm{N}$ & Soma de escores & Escore médio & $P$-value $e^{(1)}$ \\
\hline \multicolumn{5}{|c|}{$1^{\circ}$ dia } \\
\hline Água- $10 \mathrm{~mL}$ & 8 & 63,50 & 7,94 & 0,6555 \\
\hline Álcool $70 \%-10 \mathrm{~mL}$ & 8 & 72,50 & 9,06 & \\
\hline Água- $10 \mathrm{~mL}$ & 8 & 79,50 & 9,94 & 0,2374 \\
\hline${ }^{2} \mathrm{EAP} 30 \%-5 \mathrm{~mL}$ & 8 & 56,50 & 7,06 & \\
\hline Água- $10 \mathrm{~mL}$ & 8 & 88,00 & 11,00 & 0,0355 \\
\hline EAP $30 \%-10 \mathrm{~mL}$ & 8 & 48,00 & 6,00 & \\
\hline Álcool 70\% - $10 \mathrm{~mL}$ & 8 & 85,50 & 10,69 & 0,0670 \\
\hline EAP $30 \%-5 \mathrm{~mL}$ & 8 & 50,50 & 6,31 & \\
\hline Álcool $70 \%-10 \mathrm{~mL}$ & 8 & 95,50 & 11,94 & 0,0036 \\
\hline EAP $30 \%-10 \mathrm{~mL}$ & 8 & 40,50 & 5,06 & \\
\hline EAP $30 \%-5 \mathrm{~mL}$ & 8 & 76,50 & 9,56 & 0,3770 \\
\hline EAP $30 \%-10 \mathrm{~mL}$ & 8 & 59,50 & 7,44 & \\
\hline \multicolumn{5}{|c|}{$3^{\circ}$ dia } \\
\hline Água $-10 \mathrm{~mL}$ & 7 & 47,50 & 6,79 & 0,5442 \\
\hline Álcool $70 \%-10 \mathrm{~mL}$ & 7 & 57,50 & 8,21 & \\
\hline Água- $10 \mathrm{~mL}$ & 7 & 73,50 & 10,50 & 0,0412 \\
\hline EAP $30 \%-5 \mathrm{~mL}$ & 8 & 46,50 & 5,81 & \\
\hline Água- $10 \mathrm{~mL}$ & 7 & 77,00 & 11,00 & 0,0154 \\
\hline EAP $30 \%-10 \mathrm{~mL}$ & 8 & 43,00 & 5,38 & \\
\hline Álcool $70 \%-10 \mathrm{~mL}$ & 7 & 79,50 & 11,36 & 0,0065 \\
\hline EAP $30 \%-5 \mathrm{~mL}$ & 8 & 40,50 & 5,06 & \\
\hline Álcool $70 \%-10 \mathrm{~mL}$ & 7 & 82,00 & 11,71 & 0,0026 \\
\hline EAP $30 \%-10 \mathrm{~mL}$ & 8 & 38,00 & 4,75 & \\
\hline EAP $30 \%-5 \mathrm{~mL}$ & 8 & 74,00 & 9,25 & 0,5580 \\
\hline EAP $30 \%-10 \mathrm{~mL}$ & 8 & 62,00 & 7,75 & \\
\hline
\end{tabular}

(1) Probabilidade de significância da soma de escores pelo teste de Mann-Whitney, com aproximação normal às somas de escores e hipótese alternativa bilateral. ${ }^{2}$ EAP $=$ Extrato alcoólico de própolis. Fonte: Autores.

Os escores de OPG das fezes ovinas apresentaram o mesmo comportamento tanto ao sétimo dia, quanto ao vigésimo primeiro dia após o tratamento (Tabela 3). A administração de água e álcool não diferiu quanto à redução no número de OPG das fezes ovinas. A utilização de $5 \mathrm{~mL}$ de EAP 30\% reduziu a OPG das fezes em comparação aos ovinos tratados com $10 \mathrm{~mL}$ de álcool, porém não diferiu dos animais tratados com $10 \mathrm{~mL}$ de água. Todavia, a utilização de $10 \mathrm{~mL}$ de EAP $30 \%$ reduziu o número de OPG tanto em relação ao tratamento com $10 \mathrm{~mL}$ água ou com álcool $70 \%$, embora não tenha diferido com relação à administração de $5 \mathrm{~mL}$ de EAP $30 \%$.

\section{Discussão}

A solução alcoólica na concentração de $70 \%$ é geralmente utilizada para a extração do extrato de própolis. A ausência de resultado com a utilização de $10 \mathrm{~mL}$ de álcool $70 \%$ indica que sua utilização exclusiva não possui efeito sobre a carga parasitária, garantido que os efeitos do EAP são provenientes de compostos ativos da própolis.

A utilização de $5 \mathrm{~mL}$ de EAP $30 \%$ não reduziu a OPG após $1^{\circ}$ dia a administração desse tratamento. Provavelmente, a dosagem do EAP, aliada ao pouco tempo disponível para ação desse extrato, pode ter contribuído para a ineficiência de sua atuação sobre os parasitas adultos que realizavam a postura, bem como sobre o número de ovos. As concentrações de OPG ao $3^{\circ}$ e $7^{\circ}$ dia reduziram com utilização de $5 \mathrm{~mL}$ de EAP $30 \%$ em relação ao dia zero, enquanto aos 21 dias houve somente uma 
leve redução. Isso indica que, provavelmente, a administração de $5 \mathrm{~mL}$ de EAP a $30 \%$ tenha sido mais efetiva sobre o número de OPG até o $7^{\circ}$ dia, reduzindo sua eficácia no período subsequente, medida no $21^{\circ}$ dia após essa administração.

Tabela 3 - Comparativo entre os tratamentos da diferença do escore de número de ovos por grama (OPG) inicial das fezes de ovinos em relação a OPG do $7^{\circ}$ e o $21^{\circ}$ dia após a administração dos tratamentos.

\begin{tabular}{|c|c|c|c|c|}
\hline Tratamento & 1 & Soma de escores & Escore médio & $P$-value ${ }^{(1)}$ \\
\hline \multicolumn{5}{|c|}{$7^{\circ}$ dia } \\
\hline Água- $10 \mathrm{~mL}$ & $?$ & 51,50 & 7,36 & 0,9461 \\
\hline Álcool 70\%- $10 \mathrm{~mL}$ & ? & 53,50 & 7,64 & \\
\hline Água- $10 \mathrm{~mL}$ & $?$ & 70,00 & 10,00 & 0,1159 \\
\hline${ }^{2} \mathrm{EAP} 30 \%-5 \mathrm{~mL}$ & $\varepsilon$ & 50,00 & 6,25 & \\
\hline Água- $10 \mathrm{~mL}$ & $?$ & 77,00 & 11,00 & 0,0170 \\
\hline EAP $30 \%-10 \mathrm{~mL}$ & $\varepsilon$ & 43,00 & 5,38 & \\
\hline Álcool 70\%- $10 \mathrm{~mL}$ & $?$ & 77,00 & 11,00 & 0,0169 \\
\hline EAP $30 \%-5 \mathrm{~mL}$ & $\varepsilon$ & 43,00 & 5,38 & \\
\hline Álcool 70\%- $10 \mathrm{~mL}$ & $?$ & 84,00 & 12,00 & 0,0013 \\
\hline EAP $30 \%-10 \mathrm{~mL}$ & $\varepsilon$ & 36,00 & 4,50 & \\
\hline EAP 30\% - $5 \mathrm{~mL}$ & $\hat{\varepsilon}$ & 71,50 & 8,94 & 0,7504 \\
\hline EAP $30 \%-10 \mathrm{~mL}$ & $\varepsilon$ & 64,50 & 8,06 & \\
\hline \multicolumn{5}{|c|}{$21^{\circ} \mathrm{dia}$} \\
\hline Água- $10 \mathrm{~mL}$ & $?$ & 57,00 & 8,14 & 0,6041 \\
\hline Álcool $70 \%-10 \mathrm{~mL}$ & $i$ & 48,00 & 6,86 & \\
\hline Água- $10 \mathrm{~mL}$ & $?$ & 70,00 & 10,00 & 0,1172 \\
\hline EAP $30 \%-5 \mathrm{~mL}$ & $\varepsilon$ & 50,00 & 6,25 & \\
\hline Água- $10 \mathrm{~mL}$ & $?$ & 77,00 & 11,00 & 0,0173 \\
\hline EAP $30 \%-10 \mathrm{~mL}$ & $\varepsilon$ & 43,00 & 5,38 & \\
\hline Álcool 70\%- $10 \mathrm{~mL}$ & $?$ & 77,00 & 11,00 & 0,0174 \\
\hline EAP $30 \%-5 \mathrm{~mL}$ & $\varepsilon$ & 43,00 & 5,38 & \\
\hline Álcool 70\%- $10 \mathrm{~mL}$ & $?$ & 83,00 & 11,86 & 0,0020 \\
\hline EAP $30 \%-10 \mathrm{~mL}$ & $\varepsilon$ & 37,00 & 4,63 & \\
\hline EAP 30\% - $5 \mathrm{~mL}$ & $\varepsilon$ & 68,50 & 8,56 & 1,0000 \\
\hline EAP $30 \%-10 \mathrm{~mL}$ & $\varepsilon$ & 67,50 & 8,44 & \\
\hline
\end{tabular}

${ }^{1}$ Probabilidade de significância pelo teste da soma de escores de Mann-Whitney com aproximação normal às somas de escores e hipótese alternativa bilateral. ${ }^{2}$ EAP $=$ Extrato alcoólico de própolis. Fonte: Autores.

Além da maior dosagem $(10 \mathrm{~mL})$, o que pode ter contribuído para tal efeito logo após o $1^{\circ}$ dia do uso dos tratamentos, foi o modo de administração. Com a administração oral, o EAP ficou prontamente disponível para atuar sobre os parasitas, visto que, em casos de aplicação intramuscular ou subcutânea, o animal precisaria antes metabolizar o EAP para que posteriormente os compostos ativos agissem na redução da OPG.

Em estudo realizado por Sena-Lopes et al. (2018) avaliando o efeito antiparasitário de $500 \mu \mathrm{g} / \mathrm{mL}$ de própolis vermelha brasileira sobre o crescimento in vitro de Trichomonas vaginalis, os pesquisadores observaram que a própolis reduziu o crescimento de trofozoítos em 36\% nas primeiras 12 horas e causou a morte completa dos parasitas após 24 horas da aplicação. Esse fato mostra que a própolis tem capacidade de reduzir o crescimento de parasitos nas primeiras 24 horas, todavia, a ausência de efeito no presente estudo pode ser um indicativo de que a dose de $5 \mathrm{~mL}$ não foi suficiente para garantir esse resultado in vivo.

Os resultados obtidos corroboram os de Heinzen et al. (2012), que obtiveram redução de 48,48\% no percentual de OPG em $83 \%$ dos bezerros submetidos à administração oral de $10 \mathrm{~mL}$ de EAP a $30 \%$, a cada 8 horas, durante quatro dias consecutivos. A utilização de $10 \mathrm{~mL}$ de EAP também foi citada como a melhor dosagem ao testarem o EAP a $3 \%$ no controle de helmintíases de ovelhas West African (Principal et al., 2002). 
Outra evidência do efeito da própolis sobre parasitas ocorreu no estudo de Issa (2015), que administrou $250 \mathrm{mg} / \mathrm{kg} / \mathrm{dia}$ desse composto, por via oral, durante cinco dias consecutivos, verificando uma redução de 61,80\% na OPG em fezes de ratos portadores de Schistosoma mansoni, e de 58,38\% em vermes adultos, enquanto que a administração do anti-helmíntico químico praziquantel, na dose de $500 \mathrm{mg} / \mathrm{kg} / \mathrm{dia}$, por dois dias consecutivos, proporcionou uma redução de 98,38\% no OPG e 83,65\% em vermes adultos. Apesar dos anti-helmínticos químicos apresentarem um percentual mais efetivo na redução parasitária, a própolis é uma alternativa de controle natural que se torna vantajosa por reduzir a resistência dos parasitas e a transferência de resíduos aos produtos animais (Heinzen et al., 2012).

A ausência de efeito entre $5 \mathrm{~mL}$ de EAP $30 \%$ reflete que essa dosagem apresentou uma eficácia parcial, corroborando com Ferreira et al. (2013), que testaram EAP na concentração de 50\% e obtiveram uma redução in vitro de até $99,1 \%$ para Rhipicephalus Boophilus microplus, entretanto ao testarem EAP em menores concentrações a eficácia do produto reduziu para $50,9 \%$.

A ação da própolis sobre os parasitas ainda não está bem elucidada, porém, sabe-se que os flavonoides podem afetar a osmorregulação e os tecidos conjuntivos dos parasitas, bem como reduzir a eclodibilidade de ovos imaturos (Hegazi et al., 2007; Cruz-Cervantes et al., 2018). Hegazi et al. (2007) avaliaram o efeito in vitro do extrato de própolis sobre o tegumento de vermes adultos de Fasciola gigantica e observaram que a concentração de $30 \mu \mathrm{g} / \mathrm{mL}$ provocou lesão tecidual severa, de forma que, após 24 horas, não havia presença visível de tegumento nos parasitas, mas apenas uma massa de estruturas fibrosas. Estes autores ainda relataram que a perda do tegumento facilita a penetração da própolis em camadas de tecidos mais profundas, o que provoca danos maiores ao parasita.

Além disso, em termos de sanidade animal, o EAP apresenta efeitos sobre a redução de bactérias de mastite caprina (Silva et al., 2012). Morsy et al. (2016) verificaram aumento na contagem total de leucócitos, proteínas e concentrações de globulina e glicose, e diminuição da contagem de células somáticas quando administraram 3 g/dia de própolis na dieta de ovelhas. A suplementação de própolis vermelha em bezerros pré-desmamados apresentou diminuição do escore fecal, da incidência de diarreia e, consequentemente, menor gasto com medicamentos veterinários (Slanzon et al., 2019).

A redução na OPG observada nesse trabalho com a utilização de $10 \mathrm{~mL}$ de EAP a 30\%, utilizando-se própolis da região oeste do Paraná, confirma o potencial de ação da própolis sobre a carga parasitária em ovinos. Além disso, a utilização do EAP torna-se uma alternativa terapêutica complementar no controle de verminoses, principalmente em sistemas de criação agroecológicos e orgânicos (Ferreira et al., 2013; Santos et al., 2020).

\section{Conclusão}

A administração via oral, em dose única, de $10 \mathrm{~mL}$ de extrato alcoólico da própolis a 30\%, reduziu de maneira efetiva o número de ovos por grama de fezes do $1^{\circ}$ até o $21^{\circ}$ dia após o tratamento, demonstrando efeito antiparasitário. Desse modo, a utilização de extrato de própolis $30 \%$ é um método eficaz no controle da verminose de ovinos, podendo ser utilizado como um método alternativo aos produtos químicos sintéticos.

\section{Referências}

Buck, E. L., Mizubuti, I. Y., Souza, F. P., Ribeiro, R. P., Filho, A. L., Bracchini, G., \& Lopera-Barrero, N. M. (2017). Effect of propolis extract on the parasite load of Nile tilapias reared in cages. Semina: Ciências Agrárias, 38 (3), 1615-1623. https://doi.org/10.5433/1679-0359.2017v38n3p1615

Calegari, M. A., Prasniewski, A., Silva, C. D., Sado, R. Y., Maia, F., Tonial, L., \& Oldoni, T. L. (2017). Propolis from Southwest of Parana produced by selected bees: Influence of seasonality and food supplementation on antioxidant activity and phenolic profile. Anais da Academia Brasileira de Ciências, 89 (1), 45-55. https://doi.org/10.1590/0001-3765201620160499

Coelho, J., Falcao, S. I., Vale, N., Almeida-Muradian, L. B., \& Vilas-Boas, M. (2017). Phenolic composition and antioxidant activity assessment of southeastern and south Brazilian propolis. Journal of Apicultural Research, 56(1), 21-31. https://doi.org/10.1080/00218839.2016.1277602 
Cruz-Cervantes, J. A., Benavides-González, F., Sánchez-Martínez, J. G., Vázquez-Sauceda, M. D. L. L., \& Ruiz-Uribe, A. J. (2018). Propolis in aquaculture: A review of its potential. Reviews in Fisheries Science \& Aquaculture, 26(3), 337-349. https://doi.org/10.1080/23308249.2018.1424798

Ferreira, F. B. P., Pereira, M. F., Viana, R. V., Ferarrese, L., Cerquetani, J. D. A., Alberton, O., \& Gazim, Z. C. (2013). An in vitro assessment of the alcoholic extract in propolis for the control of Rhipicephalus (Boophilus) microplus. Arquivos de Ciências Veterinárias e Zoologia da UNIPAR, 16(2), $107-112$.

Garcia, R. C., de Sá, M. E. P., Langoni, H., \& Funari, S. R. C. (2004). Effect of alcohol extract of propolis on Pasteurella multocida in vitro and in rabbits. Acta Scientiarum-Animal Sciences, 26(1), 69-77.

Gharsa, H., Slama, K. B., Lozano, C., Gómez-Sanz, E., Klibi, N., Sallem, R. B., \& Torres, C. (2012). Prevalence, antibiotic resistance, virulence traits and genetic lineages of Staphylococcus aureus in healthy sheep in Tunisia. Veterinary Microbiology, 156(3-4), 367-373. https://doi.org/10.1016/j.vetmic.2011.11.009

Goncuoglu, M., Ormanci, F. S. B., Ayaz, N. D., \& Erol, I. (2010). Antibiotic resistance of Escherichia coli O157: H7 isolated from cattle and sheep. Annals of Microbiology, 60(3), 489-494. https://doi.org/10.1007/s13213-010-0074-8.

Gordon, H. M., \& Whitlock, H. V. (1939). A new technique for counting nematode eggs in sheep faeces. Journal of the Council for Scientific and Industrial Research, 12(1), 50-52.

Hegazi, A. G., Abd El Hady, F. K., \& Shalaby, H. A. (2007). An in vitro effect of propolis on adult worms of Fasciola gigantica. Veterinary Parasitology, 144(3-4), 279-286. https://doi.org/10.1016/j.vetpar.2006.10.006

Heinzen, E. L., Peixoto, E. D. M., Jardim, J. G., Garcia, R. C., Oliveira, N. T. E., \& Orsi, R. D. O. (2012). Extract of propolis in the control of helminthiasis in calves. Acta Veterinaria Brasilica, 6(1), 40-44.

IBGE. (2018). Produção da Pecuaria Municipal. Instituto Brasileiro de Geografia e Estatistica, v.44.

Issa, R.M. (2015). Schistosoma mansoni: The prophylactic and curative effects of propolis in experimentally infected mice. Rawal Medical Journal, 32, 9498.

Ítavo, C. C. B. F., Ítavo, L. C. V., Esteves, C. A. T., Sapaterro, G. A., Silva, J. A., Silva, P. C. G., \& Arco, T. F. F. S. (2019). Influence of solid residue from alcoholic extraction of brown propolis on intake, digestibility, performance, carcass and meat characteristics of lambs in feedlot. Journal Animal and Feed Sciences, 28, 149-158.

Kenyon, F., Hutchings, F., Morgan-Davies, C., van Dijk, J., \& Bartley, D. J. (2017). Worm control in livestock: bringing science to the field. Trends in parasitology, 33(9), 669-677. https://doi.org/10.1016/j.pt.2017.05.008

Lima, M., Orsi, R. D. O., Costa, G. D. M., \& Malaspina, O. (2015). Brazilian propolis production by africanized bees (Apis mellifera). Bee World, 92(3), 5868. https://doi.org/10.1080/0005772X.2015.1129229

Machado, B., Pulcino, T. N., Silva, A. L., Tadeu, D., Melo, R. G. S., \& Mendonça, I. G. (2016). Propolis as an alternative in prevention and control of dental cavity. Journal Apither, 1(2), 47-50. https://doi.org/10.5455/ja.20160726115117

Monroy, Y. M., Rodrigues, R. A., Rodrigues, M. V., \& Cabral, F. A. (2018). Fractionation of ethanolic and hydroalcoholic extracts of green propolis using supercritical carbon dioxide as an anti-solvent to obtain artepillin rich-extract. The Journal of Supercritical Fluids, 138, 167-173. https://doi.org/10.1016/j.supflu.2018.04.016

Morsy, A. S., Soltan, Y. A., Sallam, S. M., Alencar, S. M., \& Abdalla, A. L. (2016). Impact of Brazilian red propolis extract on blood metabolites, milk production, and lamb performance of Santa Inês ewes. Tropical Animal Health and Production, 48(5), 1043-1050.

Morsy, A. S., Abdalla, A. L., Soltan, Y. A., Sallam, S. M., El-Azrak, K. E. D. M., Louvandini, H., \& Alencar, S. M. (2013). Effect of Brazilian red propolis administration on hematological, biochemical variables and parasitic response of Santa Inês ewes during and after flushing period. Tropical Animal Health and Production, 45(7), 1609-1618. https://doi.org/10.1007/s11250-013-0406-3

Principal, J., Hernández, I., D’aubeterre, R. Gervacio R. J. (2002). Efficacy of propolis on the control of naturally infested sheep helminthiasis. Revista Cientifica, 12(2), 604-607.

Salkova, D. S., Panayotova-Pencheva, M. S., Movsesyan, S. O. (2015). Some bee products as antiparasitic remedies. Russian Journal of Parasitology, 619, $75-78$.

Santos, H. C. D., Vieira, D. S., Yamamoto, S. M., Costa, M. M., Sá, M. C., Silva, E., \& Silva, T. (2019). Antimicrobial activity of propolis extract fractions against Staphylococcus spp. isolated from goat mastitis. Pesquisa Veterinária Brasileira, 39(12) 954-960. https://doi.org/10.1590/1678-5150-PVB-5940

Sargison, N. D. (2020). The critical importance of planned small ruminant livestock health and production in addressing global challenges surrounding food production and poverty alleviation. New Zealand Veterinary Journal, 68(3), 136-144. https://doi.org/10.1080/00480169.2020.1719373

Sena-Lopes, A., Bezerra, F. S. B., das Neves, R. N., Pinho, R. B., Silva, M. T. D. O., Savegnago, L., \& Borsuk, S. (2018). Chemical composition, immunostimulatory, cytotoxic and antiparasitic activities of the essential oil from Brazilian red propolis. PLoS One, $13(2)$, e0191797. https://doi.org/10.1371/journal.pone.0191797

Shabana, I. I., \& Al-Enazi, A. T. (2020). Investigation of plasmid-mediated resistance in E. coli isolated from healthy and diarrheic sheep and goats. Saudi Journal of Biological Sciences, 27(3), 788-796. https://doi.org/10.1016/j.sjbs.2020.01.009

Shedeed, H. A., Farrag, B., Elwakeel, E. A., Abd El-Hamid, I. S., \& El-Rayes, M. A. H. (2019). Propolis supplementation improved productivity, oxidative status, and immune response of Barki ewes and lambs. Veterinary World, 12(6), 834. https://doi.org/10.14202/vetworld.2019.834-843 
Silva, C. S. R., Barreto, C. L. P., Peixoto, R. M., Mota, R. A., Ribeiro, M. F., \& Costa, M. M. (2012). Antibacterial effect of Brazilian brown propolis in different solvents against staphylococcus spp. Isolated from caprine mastitis. Ciência Animal Brasileira, 13(2), 247-251.

Slanzon, G. S., Toledo, A. F., Silva, A. P., Coelho, M. G., da Silva, M. D., Cezar, A. M., \& Bittar, C. M. M. (2019). Red propolis as an additive for preweaned dairy calves: Effect on growth performance, health, and selected blood parameters. Journal of Dairy Science, 102(10), 8952-8962.

Snedecor, G.W., \& Cochran, W. G. (1989). Statistical methods. 8.ed. Lowa: State Univ. Press Iowa. 